\title{
A simple method for constructing magnetic Escherichia coli
}

\section{Mengyi Sun, Yong-jun Lu*}

3

4 School of Life Sciences and Biomedical Center, Sun Yat-sen University, No. 135, Xingang Xi

5 Road, Guangzhou, 510275, P. R. China

6

7 Mengyi Sun, sunmengyi2014@gmail.com

*Corresponding author: Tel.: +86-020-84110778, Fax: +86-020-84036215, e-mail:

$9 \quad$ luyj@mail.sysu.edu.cn

10

11

12

13

14

15

16

17

18

19

20

21

22

23

24

25

26

27

28

29 


\section{Abstract}

32 Magnetic force can serve as an ideal way to control the spatial behavior of microorganisms, 33 because of its flexibility and penetrability. By incubation with the biocompatible compound, 34 ammonium ferric citrate, as an iron source, we magnetized Escherichia coli, the most 35 programmable chassis in synthetic biology. To enhance the magnetization efficiency, the ferritin 36 protein, FtnA, from E. coli was cloned and overexpressed in strain BL21(DE3). The 37 magnetization effect was observed within $30 \mathrm{~min}$ after harvest of bacteria, and the concentration 38 of ammonium ferric acid used could be as low as $0.5 \mathrm{mM}$. Using different shapes of magnetic 39 fields, different patterns could be generated easily. Our method may set up the foundation for a 40 rational design of spatial structure of cell communities, which is important for their actual 41 application.

42

43 Keywords: Ammonium ferric citrate; E. coli; FtnA; Magnetization 


\section{Introduction}

63

Controlling the movement and location of cells is one of the most important topics in synthetic biology. Pattern formation, synthetic consortia and the novel cell array sensor heavily rely on the precise spatial arrangement of cells (Melamed et al. 2012; Brenner et al. 2008). The traditional method relied on modifying the chemotaxis mechanism of cells, which was both slow and irreversible (Goldberg et al. 2009). Recently, rapid control of cells has been achieved by introducing light controllable proteins into organisms, hence the name optogenetics (Herrou and Crosson 2011). However, the heat effect of light prevented its use in prolonged control. Furthermore, the low penetrability of light hindered its application. Compared with chemical and light control, magnetic force is safe, fast, reversible, highly penetrative and easy to design (Pankhurst et al. 2003), and thus would be an ideal method for controlling cells if the cells could be magnetized. Therefore, several methods to magnetize cells have been developed, including methods that rely on artificial synthesis of magnetic nanoparticles (Cho et al. 2012), and transferring the cassette controlling magnetosome synthesis in Magnetospirillum gryphiswaldense into the related species, Rhodospirillum rubrum (Kolinko et al. 2014). However, these methods are both laborious and hard to generalize. In 2011, Silver's group (Nishida and Silver 2012) reported a simple method for magnetizing budding yeast, by incubation with ferric citrate and introduction of a ferritin protein.

So far, no simple method for magnetizing the most programmable chassis organism in synthetic biology has been reported. Here, we extended Silver's method to Escherichia coli. After incubation with ammonium ferric citrate, we showed the magnetization by the formation of defined patterns under strong magnets. By cloning and overexpressing FtnA, a ferritin protein that helps store iron in E. coli (Bitoun et al. 2008), we showed that the magnetization effect 
86 could be amplified even under a low concentration of $\mathrm{Fe}^{3+}$. Using different shapes of magnets,

87 we also showed the flexibility of patterns formed by bacteria. Our method provides a versatile 88 tool for spatial control of E. coli, which may aid the development of synthetic biology.

\section{Methods}

91

\section{Strains, culture medium and conditions}

93

94 The E. coli strains used for cloning FtnA, plasmid construction and the attraction test were K12, 95 DH5 $\alpha$ and BL21(DE3), respectively. The medium used was LB. Ampicillin was added when necessary. Except for induction, the bacteria were cultured at $37^{\circ} \mathrm{C}$ with agitation at $220 \mathrm{rpm}$.

Plasmid construction

The ftnA gene was amplified from strain K12 and cloned into plasmid pUC18. A mutation was introduced to eliminate the NdeI restriction site in the original $f \operatorname{tn} A$ sequence using the using the NdeI and BamHI restriction sites (Fig. 1).

Insert Figure 1

105

Growth test

Experiments were performed at $37^{\circ} \mathrm{C}$ with agitation at $220 \mathrm{rpm} . \mathrm{OD}_{600}$ values were measured at several time points, and converted into dry weight. The experiments were performed in triplicate.

110

\section{Magnetic cells preparation}

112 
113 For attraction tests without plasmid induction, bacteria were cultured in LB medium inoculated

114 with $20 \mathrm{mM} \mathrm{Fe}^{3+}$ derived from ammonium ferric citrate. Cells were harvested after $24 \mathrm{~h}$ and

115 washed several times, then pelleted by centrifugation and resuspended in double-distilled $\mathrm{H}_{2} \mathrm{O}$.

116 For induction experiments, cells were exposed to $20 \mathrm{mM} \mathrm{Fe}^{3+}$ and induced with $1 \mathrm{mM}$ isopropyl

$117 \beta$-D-1-thiogalactopyranoside (IPTG) at $25^{\circ} \mathrm{C}$ for $24 \mathrm{~h}$, and then harvested as described above.

119 Attraction test

120

121 Three milliliters of cell suspension were added to $3.5-\mathrm{cm}$ dishes. For characterizing the magnetization effect, a circular Rubidium iron boron magnet (2-cm in diameter) was put underneath each dish, representing a magnetic field of $1.2 \mathrm{~T}$ near the magnet edge. For generating different patterns, different shapes of magnets were used, accordingly. Photographs were taken at appropriate time points.

\section{Results and Discussion}

\section{Effect of ammonium ferric citrate on $E$. coli growth}

131 To obtain magnetic $E$. coli as much as possible, we used ammonium ferric citrate, since citrate

132 has been reported as an effective microorganism siderophore for iron absorption (Guerinot et al.

133 1990). We compared the growth of E. coli under high iron concentration (20 mM ammonium

134 ferric citrate added from a fresh stock of $500 \mathrm{mM}$ ) and normal conditions by measuring the

$135 \mathrm{OD}_{600}$ at several time points after inoculation. As shown in Fig. 2, no significant difference was 136 observed between the control group and the experimental group, demonstrating that ammonium

137 ferric citrate is not toxic to E. coli cells. Hence, ammonium ferric citrate could serve as the iron 138 source without decreasing the amount of E. coli harvested. This enabled us to easily maintain a 
139 population that is much larger than any other chassis organism for a synthetic cell-cell

140 community (Youk and Lim 2014).

\section{Insert Figure 2}

142 Magnetization of $E$. coli by incubation with ammonium ferric citrate

144 Next, we tested whether E. coli could be magnetized by incubation with ammonium ferric

145 citrate. Cells were harvested $24 \mathrm{~h}$ after inoculation. As shown in Fig. 3a, cells harvested from

146 cultures containing $20 \mathrm{mM} \mathrm{Fe}{ }^{3+}$ formed a white circle where the magnetic force was strongest,

147 near the edge of the magnet. No noticeable cluster of E. coli was formed in the control group.

148 This result demonstrated that incubation with ammonium ferric citrate indeed magnetized $E$.

149 coli. The short time and ease of preparation of the magnetic E. coli enabled a massive 150 experiment to be performed in a short time.

\section{The time course of the attraction phenomena}

154 As shown in Fig. 3b, the attraction ring appeared $1 \mathrm{~h}$ after putting the magnet under the culture 155 plate, and the pattern was stable after $2 \mathrm{~h}$. Note that the pattern was formed in a liquid 156 environment, which could not be accomplished by the traditional method with the chemotaxis

157 mechanism, because the chemicals would quickly become uniform by diffusion. The E. coli 158 could respond to the magnetic fields so quickly, because of the high efficiency of iron absorption 159 of bacteria (Andrews et al. 2003). It is also well known that E. coli can express ferritin, a 160 ubiquitous iron storage protein that is conserved from bacterium to human. This protein becomes 161 paramagnetic when iron is incorporated (Bauminger and Nowik 1989). Note that in our 162 experiment, the time required to form the attraction ring was slightly longer than that in Silver's 163 experiment using yeast, probably because $E$. coli cells are much lighter than yeast cells (Nishida 164 and Silver 2012).

\section{Insert Figure 3}


168 To achieve a higher magnetization efficiency of $E$. coli, we cloned and overexpressed FtnA, a

169 ferritin protein, in E. coli to enhance the efficiency of ferrite incorporation. This protein also

170 increases the iron stores, which could be used during iron deficiency (Abdul-Tehrani et al. 1999).

171 As shown in Fig. 4, the attraction ring was formed within $30 \mathrm{~min}$ in the experimental group,

172 while in the control group no observable ring was formed in such a short time. Note that the

173 concentration of $\mathrm{Fe}^{3+}$ used could be as low as $0.5 \mathrm{mM}$. Thus, the introduction of FtnA

174 significantly increased the magnetization efficiency. This result also revealed a remarkable

175 advantage of our system: the concentration of the iron source used could be as low as $0.5 \mathrm{mM}$,

176 which was much lower than that required for magnetizing yeast (Nishida and Silver 2012).

\section{Insert Figure 4}

\section{The flexibility of the pattern formed by magnets of different shapes}

180 To show the flexibility of the cell pattern formation, we used different shapes of magnets to

181 induce different pattern shapes. As shown in Fig. 5, a rectangular shape and small double circles

182 were formed under the attraction of rectangular magnets and small circular magnets,

183 respectively. Interestingly, our method generated accurate pattern edges comparable to the

184 synthetic edge detection circuit in E. coli (Tabor et al. 2009), without complex genetic design or

185 mathematical modeling.

$186 \underline{\text { Insert Figure } 5}$

\section{Conclusion}

189 Here, we established a simple method for producing magnetized E. coli by incubation with

190 ammonium ferric citrate. The attraction ring pattern could be formed within $1 \mathrm{~h}$. Overexpression

191 of FtnA in E. coli significantly improved the magnetization effect. Furthermore, different

192 patterns could easily be generated by different shapes of magnets, demonstrating the flexibility 
193 of our method. This method sheds light on using the magnetic force to control the most

194 programmable microorganism, E. coli, in synthetic biology.

195

196 Acknowledgments

197

198 We thank Dr. He Lei for his assistance with graphing, and members of XiongLei He's lab for

199 valuable suggestions and discussion. This work was supported by NSFC (No. J1310025).

200 The authors declare no conflict of interest.

201

202 References

203

204 Abdul-Tehrani H, Hudson AJ, Chang YS, Timms AR, Hawkins C, Williams JM et al (1999)

205 Ferritin mutants of Escherichia coli are iron deficient and growth impaired, and fur mutants are 206 iron deficient. J Bacteriol 181:1415-1428

207

208 Andrews SC, Robinson AK, Rodríguez-Quiñones F (2003) Bacterial iron homeostasis. FEMS 209 Microbiol Rev 215-237

210

211 Bauminger ER, Nowik I (1989) Magnetism in plant and mammalian ferritin. Hyperfine Interact $212 \quad 50: 489-497$

213

214 Bitoun JP, Wu G, Ding H (2008) Escherichia coli FtnA acts as an iron buffer for re-assembly of

215 iron-sulfur clusters in response to hydrogen peroxide stress. Biometals 21:693-703 doi: $216 \quad 10.1007 / \mathrm{s} 10534-008-9154-7$

218 Brenner K, You L, Arnold FH (2008) Engineering microbial consortia: a new frontier in 219 synthetic biology. Trends Biotechnol 26:483-489 doi: 10.1016/j.tibtech.2008.05.004 
221 Cho MH, Lee EJ, Son M, Lee JH, Yoo D, Kim JW et al (2012) A magnetic switch for the control 222 of cell death signalling in in vitro and in vivo systems. Nat Mater 11:1038-1043 doi: $223 \quad 10.1038 /$ nmat3430

224

Goldberg SD, Derr P, DeGrado WF, Goulian M (2009) Engineered single- and multi-cell chemotaxis pathways in E. coli. Mol Syst Biol 5:283 doi: 10.1038/msb.2009.41

Guerinot ML, Meidl EJ, Plessner O (1990) Citrate as a siderophore in Bradyrhizobium japonicum. J Bacteriol 172:3298-3303

230

Pankhurst QA, Connolly J, Jones SK, Dobson J (2003) Applications of magnetic nanoparticles in

Herrou J, Crosson S (2011) Function, structure and mechanism of bacterial photosensory LOV 235 proteins. Nat Rev Microbiol 9:713-723

Kolinko I, Lohße A, Borg S, Raschdorf O, Jogler C, Tu Q et al (2014) Biosynthesis of magnetic nanostructures in a foreign organism by transfer of bacterial magnetosome gene clusters. Nat Nanotechnol 9:193-197 doi: 10.1038/nnano.2014.13

Melamed S, Elad T, Belkin S (2012) Microbial sensor cell arrays. Curr Opin Biotechnol 23:2-8 doi: 10.1016/j.copbio.2011.11.024

244 Nishida K, Silver PA (2012) Induction of biogenic magnetization and redox control by a 245 component of the target of rapamycin complex 1 signaling pathway. PLoS biology 10:e1001269 246 doi: 10.1371/journal.pbio.1001269 
248 Tabor JJ, Salis HM, Simpson ZB, Chevalier AA, Levskaya A, Marcotte EM et al (2009) A

249 synthetic genetic edge detection program. Cell 137:1272-1281

250

251 Youk H, Lim WA (2014) Secreting and sensing the same molecule allows cells to achieve

252 versatile social behaviors. Science 343:1242782

253

\section{Figure Legends}

255

256 Fig. 1 The map of plasmid pET-22b+-FtnA, which was constructed by inserting the FtnA coding 257 sequence immediately after the ribosomal binding site

260 Fig. 2 Growth test of E. coli inoculated with ammonium ferric acid. The $\mathrm{OD}_{600}$ values were 261 measured at 3, 5, 7 and $12 \mathrm{~h}$. Experiments were performed in triplicate

264 Fig. 3 The test of the magnetization effect of inoculation with ammonium ferric acid by attraction ring formation. Bacteria resuspended in water were added to $3.5-\mathrm{cm}$ blank dishes. A circular magnet was placed underneath each dish to attract magnetized bacteria. (a) The attraction ring was formed only in the group inoculated with ammonium ferric citrate. (b) A time course of the attraction ring formation

Fig. 4 Overexpressing FtnA improves the magnetization effect

273 Fig. 5 Different attraction rings with a sharp edge under the action of different magnet shapes 


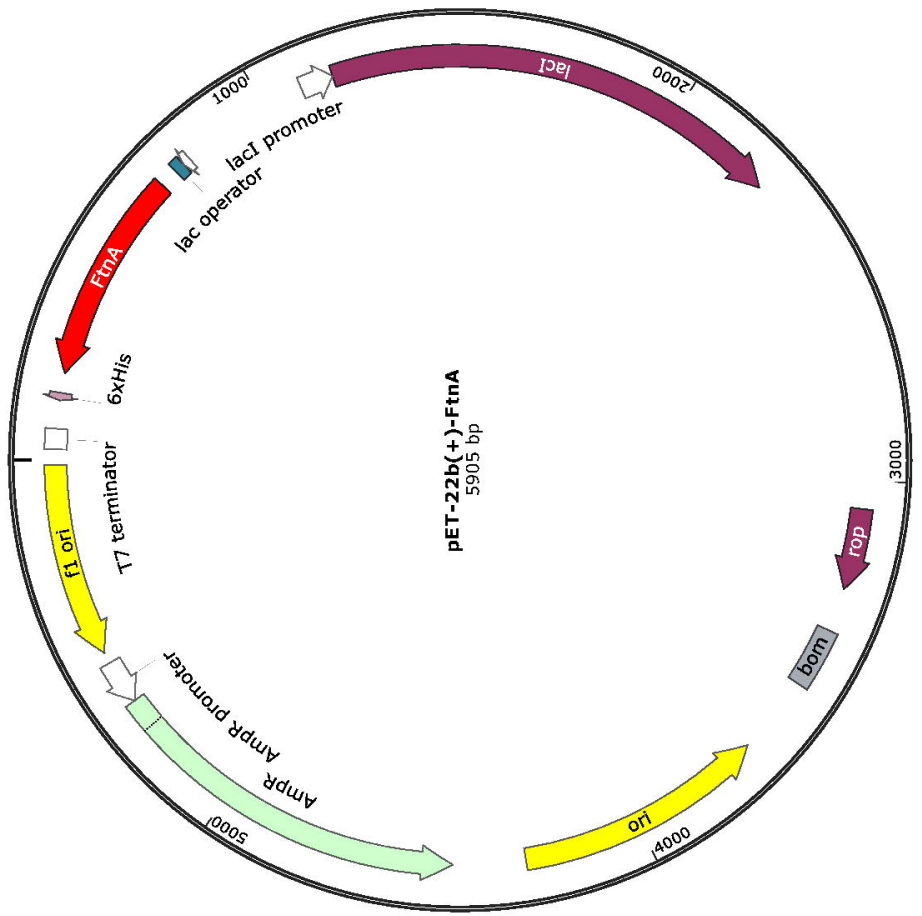


1.6

1.2

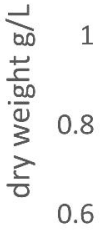

0.4

0.2

0

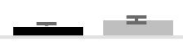

$3 h$

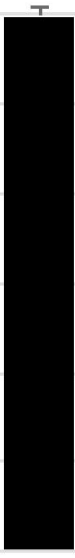

$5 h$ 포

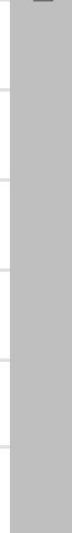

$7 h$

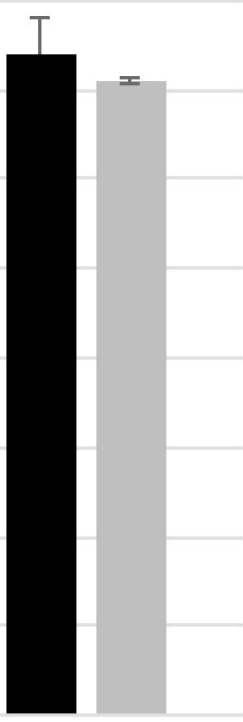

$12 \mathrm{~h}$

- control $20 \mathrm{mM} \mathrm{Fe}(3+)$ added 


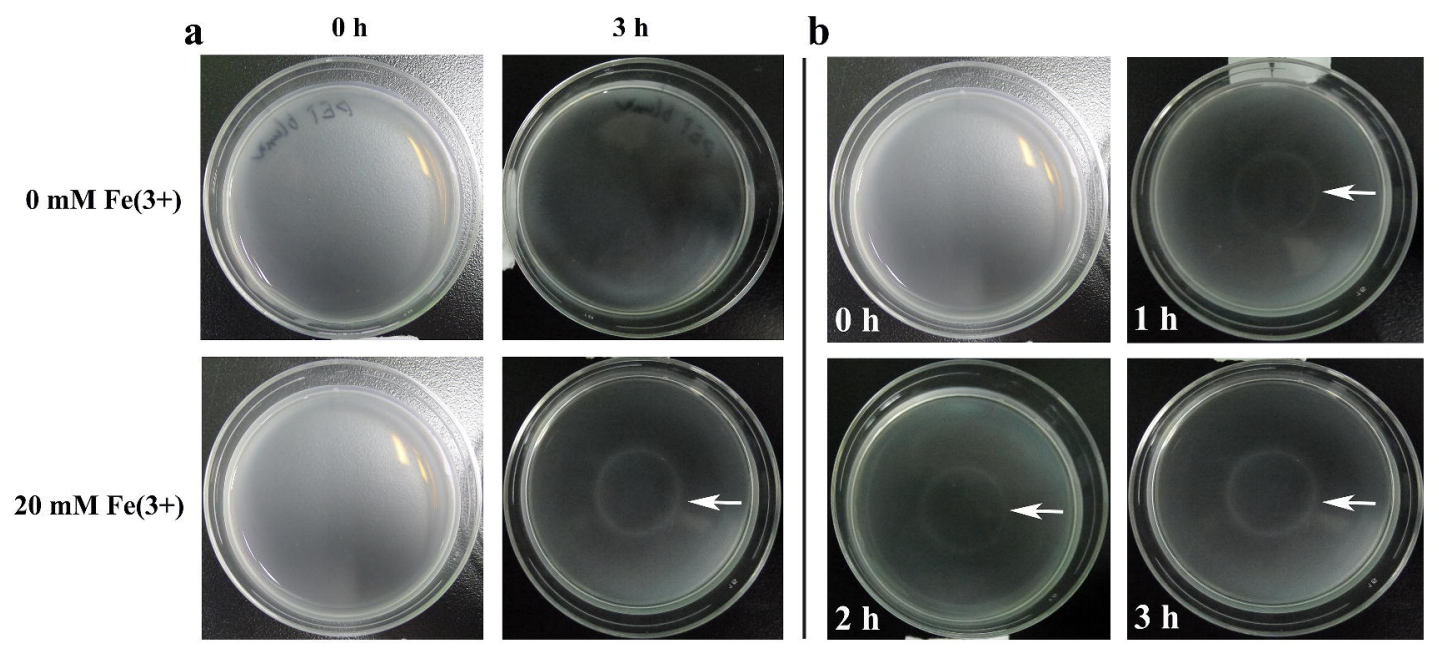


( ) h

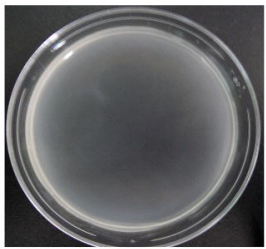

pET-22b $(+)$

pET-22b(+)-FtnA

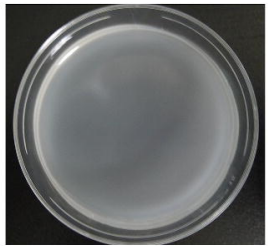

$0.5 \mathrm{~h}$
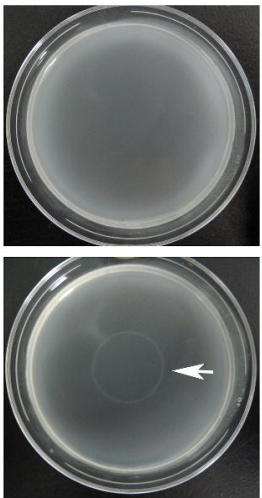


\section{Magnets}
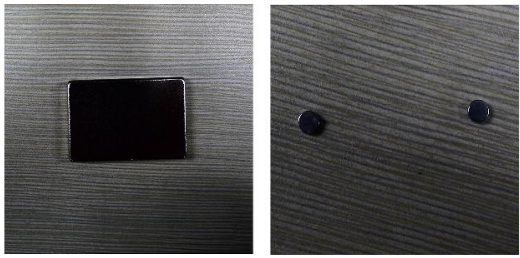

Patterns
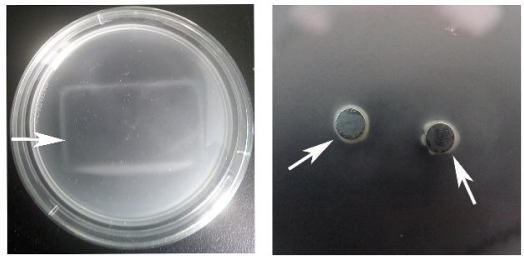